\title{
Occipitofrontal Diameter
}

National Cancer Institute

\section{Source}

National Cancer Institute. Occipitofrontal Diameter. NCI Thesaurus. Code C124481.

A measurement of the skull diameter, measured from the external occipital

protuberance to the most prominent point of the frontal bone in the midline. 